\title{
Aprendizaje basado en proyectos en los laboratorios de comunicaciones digitales.
}

\author{
Antonio Soriano-Asensi ${ }^{1}$, Jaume Segura ${ }^{1}$, Carmen Botella ${ }^{1}$, Joaquín \\ Pérez $^{1}$ y Santiago Felici i Castell ${ }^{1}$ \\ ${ }^{1}$ Departament d'Informàtica. Universitat de València. \\ Av. de la Universitat s/n, 46100 Burjassot (SPAIN)
}

\begin{abstract}
Digital Communications and Signal Processing for Communications have been traditionally taught from a theoretical point of view. The complexity and cost of radio-frequency systems hampers the realization of practical experiences in the laboratories of Communications. However, current Academic Accreditation includes the dimension of "what students are expected to be able to do". The decrease of the gap between standard simulated laboratory sessions and more practical and realistic approaches is therefore required. In this paper, we introduce an active learning methodology we have followed in order to introduce practical aspects related to Digital Communications and Signal Processing for Communications courses via low-cost software defined radio devices. The proposed strategy gives the students a key role in their learning process, which has contributed to improve the engagement of both master and under-graduate students. As an example, the laboratory activities the students did based on the IEEE 802.11 standard are presented, and recommendations for practice are given.
\end{abstract}

Keywords: SDR, Digital Communications, Signal Processing, HackRF One.

\footnotetext{
Resumen

Las Comunicaciones Digitales y el Procesado de Señal para Comunicaciones se enseñan tradicionalmente desde un punto de vista teórico. La complejidad y el coste de los sistemas de radiofrecuencia complica mucho la realización de experiencias prácticas en los laboratorios de Comunicaciones. Sin embargo, la Acreditación Académica actual incluye la dimensión
}

\footnotetext{
* Este trabajo ha sido parcialmente financiado por el Ministerio de Economía a través del proyecto BIA2016-76957-C3-1-R y por el "Servei Formació Permanent i Innovació Educativa" de la Universitat de Valencia a través del proyecto UV-SFPIE-RMD18-841566
} 
de "lo que se espera que los estudiantes sean capaces de hacer". Es por tanto necesario reducir la brecha entre las sesiones de laboratorio basadas en simulaciones y nuevos enfoques más aplicados y realistas. En este trabajo, se presenta la metodología que hemos seguido para introducir los aspectos prácticos relacionados con las Comunicaciones Digitales y el Procesado de Señal a través de dispositivos de radio definida por software de bajo coste. La estrategia propuesta pone a los estudiantes en el centro de su proceso de aprendizaje, lo que ha contribuido a mejorar su implicación en el desarrollo del laboratorio tanto en los estudiantes de máster y grado. A modo de ejemplo se presentan las actividades de laboratorio en base al estándar IEEE 802.11, a partir de la experiencia llevada a cabo se proporcionan recomendaciones para la realización de éstas actividades prácticas.

Keywords: SDR, Comunicaciones Digitales, Procesado de Señal, HackRF One.

\section{Introducción}

Los grados de Ingeniería de Telecomunicación incluyen la materia de Comunicaciones Digitales como uno de los ejes centrales de sus planes de estudios. En el caso de la Escola Tècnica Superior d'Enginyeria de la Universitat de València (ETSE-UV), las Comunicaciones Digitales y el Procesado de Señales para Comunicaciones representan un $20 \%$ (24 unidades del Sistema Europeo de Transferencia de Créditos (ECTS)) de las asignaturas obligatorias y un $10 \%$ del Grado de Ingeniería Telemática (GIT) (240 ECTS). Tradicionalmente, estos bloques centrales se enseñan desde un punto de vista teórico, utilizando los elementos básicos de los sistemas de comunicación como argumento.

Los cursos de Comunicaciones Digitales y Procesado de Señal para Comunicaciones normalmente incluyen sesiones de laboratorio estándar donde los estudiantes pueden simular partes del sistema usando interfaces Matlab, Python o C. Desde nuestro punto de vista, aquí surgen dos problemas. Por un lado, algunos de los aspectos prácticos de la implementación deben simplificarse o ignorarse debido a la naturaleza de la simulación en sí y a las limitaciones de tiempo, como es el caso de imperfecciones del canal o los problemas de tiempo. Por otro lado, los Títulos Académicos tienen como objetivo la obtención de una acreditación de calidad, como la certificada por el Consejo de Acreditación de Ingeniería y Tecnología (ABET) o la Red Europea para la Acreditación de la Enseñanza de la Ingeniería (ENAEE). Estas entidades, más que centrarse en los contenidos técnicos de las titulaciones, evalúan los Objetivos Educativos del Programa y los Resultados Estudiantiles, que incluyen los conocimientos, habilidades y comportamientos que se adquieren a través del programa. Lo que es más, la dimensión de "lo que se espera que los estudiantes sean capaces de hacer"se incluye en la evaluación. Se plantea entonces en este artículo, como necesidad de la innovación, disminuir la brecha entre el conocimiento teórico explicado en clase y las aplicaciones prácticas del entorno profesional, que, al final, es el objetivo del Grado Académico.

El concepto de Software Defined Radio (SDR) (Mitola 1992) establece un nuevo paradigma educativo en el campo de las telecomunicaciones, que permite implementar muchos componentes de los sistemas de radiocomunicaciones utilizando programas 
informáticos que, además, pueden ser reconfigurados en tiempo real. En los trabajos (El-Hajjar y col. 2014; Jimenez y col. 2017; Stewart y col. 2015) se abordan los beneficios, retos y casos de uso de las plataformas SDR en la enseñanza de las comunicaciones y el procesado de señales. Los estándares como WiFi (802.11) (IEEE 2016) o Digital Video Broadcasting - Terrestrial (DVB-T) (ETSI 2009) son muy útiles para este desarrollo metodológico, ya que sus bloques funcionales muestran una buena superposición con los contenidos de los cursos relacionados con esta propuesta. Desde el curso 2015-2016 estamos llevando a cabo varias iniciativas de innovación docente (Segura y col. 2016; Segura y col. 2017), con el propósito de introducir diferentes plataformas SDR en las titulación de GIT y también en el Máster en Ingeniería de Telecomunicación de la Universitat de València (MITUV). Siguendo con esa línea de innovación, en este trabajo se propone una metodología de Aprendizaje Basado en Proyectos (ABP) para mejorar la motivación del estudiante en el aprendizaje de aspectos prácticos, relacionados con las Comunicaciones Digitales y el Procesado de Señales a través de dispositivos de SDR de bajo coste, lo que ha demostrado mejorar la participación de estudiantes de máster y grado.

El resto del artículo se organiza como sigue: en la sección 2 se detallan los objetivos planteados en la actividad de innovación docente. El desarrollo de la innovación basada en ABP se explica en la sección 3. Los resultados obtenidos con la experiencia de ABP y su traslación a los laboratorios en GIT se discuten en la sección 4. El resultado de la innovación, en el que se cuantifica el impacto de la actividad realizada en la motivación de los estudiantes de GIT se presenta en la sección 5, y en la sección 6 se resume lo aprendido en esta experiencia.

\section{Objetivos}

Desde el curso académico 2015-16 hemos llevado a cabo varias propuestas de innovación docente, en base al análisis de los requisitos del desarrollo teórico-práctico de los laboratorios para las diferentes asignaturas a nivel de grado y máster. El objetivo fundamental de estas actividades de innovación es mejorar la conexión entre los aspectos teóricos y prácticos en las asignaturas relacionadas con las Comunicaciones Digitales y el Procesado de Señales. La experiencia previa, (Segura y col. 2017), ha probado la utilidad de las prácticas de laboratorio basadas en SDR para reducir la tasa de abandono de los estudiantes. Además son los propios alumnos los que expresan la necesidad de aumentar el uso de estos dispositivos en las prácticas de laboratorio (Segura y col. 2016).

Los objetivos específicos de la experiencia planteada en este trabajo son:

- Proponer experiencias prácticas más próximas a las aplicaciones reales que tendrán que emplear los estudiantes cuando trabajen como ingenieros.

- Elaboración de material docente que promueva la implicación del estudiante en su formación académica.

- Cuantificación objetiva de la motivación de los estudiantes en las sesiones de laboratorio.

Para la consecución de estos objetivos, con los estudiantes de máster MITUV se ha planteado una estrategia basada en ABP. Se les ha proporcionado todo el material 
necesario, y se les propuesto que implementen un banco de pruebas para la evaluación de un sistema de comunicación basado en el protocolo 802.11 (Wifi). Como resultado de este trabajo, se les ha pedido que elaboren un documento que sirva de guía a otro ingeniero para poner en funcionamiento el banco de pruebas y para la realización de las medidas. El documento elaborado por los estudiantes de máster MITUV será empleado por los estudiantes de GIT para la comprobación de las mismas medidas. El propósito es que la experiencia de los estudiantes de máster sirva de realimentación en el proceso formativo de los estudiantes de grado.

La forma en que se plantea la experiencia permite que los estudiantes de máster, con mayor formación, tengan la ocasión de trabajar con mayor profundidad los detalles sobre la implementación del protocolo IEEE 802.11. Mientras que con los estudiantes de GIT se plantea una experiencia más guiada. Lo que se espera con la elaboración del material para el grado a partir del ABP realizado en el máster es que la guía de prácticas resulte más asequible al estudiante que si la preparase un profesor.

Finalmente, se pretende cuantificar la contribución de la experiencia llevada a cabo en la implicación de los estudiantes de GIT. Para evaluar esta implicación se ha realizado una encuesta, basada en una encuesta sobre motivación de los estudiantes desarrollada por el departamento de psicología de la UV (García-Ros y col. 2017; Schaufeli y Bakker 2004). En ella se evalúan tres aspectos relacionados con la motivación de los estudiantes: energía, dedicación y absorción.

\section{Desarrollo de la innovación}

El ABP es un estilo de aprendizaje activo que coloca al estudiante en el centro del proceso de aprendizaje. La exploración activa de los retos del mundo real permite a los estudiantes adquirir un conocimiento más profundo. El proceso de aprendizaje en $\mathrm{ABP}$ es dirigido por los estudiantes, mientras que el papel del profesor es guiar la investigación de los estudiantes proponiendo preguntas y tareas que les ayuden a resolver el problema propuesto. Se espera que el profesor presente el problema y proporcione a los estudiantes los materiales y la documentación necesarios para iniciar el proyecto. A medida que avanza la actividad, los estudiantes asumen el papel principal del proyecto.

\section{Preparación del proyecto}

El ABP se implementó en un curso de máster debido a la mayor experiencia de los estudiantes en comparación con los de grado. Además, el número de estudiantes de máster por curso (normalmente de 8 a 10) es menor que los 16 o 20 estudiantes por grupo de laboratorio en los cursos de grado. Los estudiantes de máster se organizaron en parejas y se les pidió a todos que diseñaran un banco de pruebas para evaluar el rendimiento de un sistema basado en el protocolo IEEE 802.11. Se les proporcionó dos HackRF One por grupo, una máquina virtual Ubuntu 16.04 (VM) con GNU Radio y el enlace al proyecto gr-ieee802-11 ${ }^{1}$ GitHub con la implementación del estándar IEEE 802.11p, que se detalla en (Bloessl y col. 2018). Durante el proyecto, los estudiantes tuvieron acceso al laboratorio de comunicaciones, donde había un puesto

\footnotetext{
${ }^{1}$ https://github.com/bastibl/gr-ieee802-11
} 


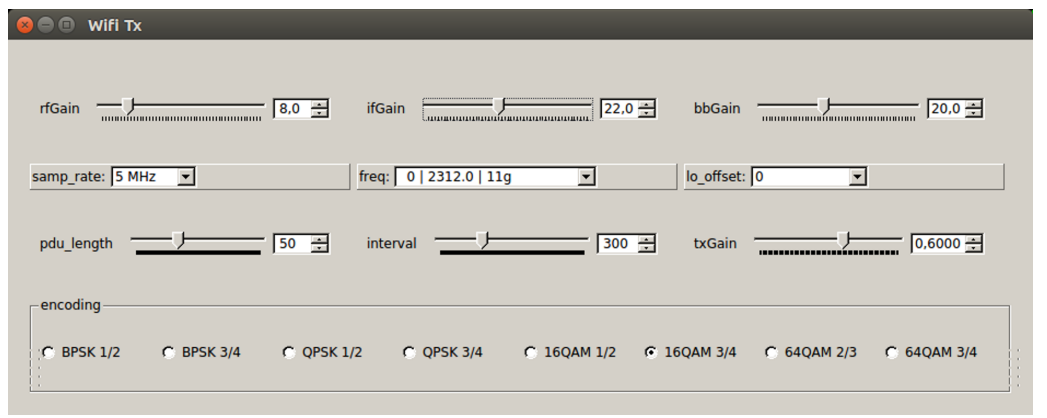

Fig. 1: Imagen de la GUI del transmisor, con los controles que permiten elegir las características de la transmisión.

de trabajo con un ordenador, que tenía el mismo software que estaba instalado en la máquina virtual. El lugar de trabajo era compartido por todos los grupos, por lo que tuvieron que organizarse para poder acceder al laboratorio. La VM permitió a los estudiantes realizar parte de la actividad en casa, mientras que el lugar de trabajo en el laboratorio de comunicaciones les permitió probar sus avances con el hardware SDR.

\section{Presentación del proyecto y tareas iniciales}

Se esperaba que los estudiantes realizaran parte de su investigación fuera de las horas lectivas, mientras que el tiempo en clase se dedicaba a comprobar la evolución de cada grupo y a ayudarles a continuar con su trabajo. La actividad requirió tres sesiones de clase (tres horas cada una). La primera sesión se utilizó para presentar el proyecto a los estudiantes. Se les preesntó la VM, el proyecto GitHub, y su documentación citando a (Bloessl y col. 2018). El proyecto SDR descrito en (Bloessl y col. 2018) está basado en dispositivos USRP, mientras que se esperaba que el proyecto en este caso se realizara con HackRF One. En esta primera sesión el profesor instruyó a los alumnos sobre las diferencias entre el USRP y el HackRF One. La tarea propuesta a los estudiantes en esta primera sesión fue adaptar el proyecto gr-ieee802-11 para ser utilizado con el HackRF One.

Las Interfaces Gráficas de Usuario (GUI) resultantes desarrolladas por los estudiantes para el transmisor y el receptor se muestran en las Fig. 1 y Fig. 2, respectivamente. Los controles de éstas GUIs permitieron a los estudiantes ajustar los parámetros del transmisor y del receptor para cada esquema de modulación. Como resultado de esta actividad los estudiantes pudieron fijar las ganancias del transmisor y del receptor HackRF One. El proyecto gr-ieee802-11 permite establecer la frecuencia de muestreo en $5 \mathrm{MHz}$ o $10 \mathrm{MHz}$. Pero, considerando que la frecuencia máxima de muestreo del HackRF One es de $10 \mathrm{MHz}$ y con el fin de reducir los requerimientos de computación del receptor, los estudiantes concluyeron que era más apropiado establecer la frecuencia de muestreo en $5 \mathrm{MHz}$. Los estudiantes también evaluaron cuál era el canal más adecuado para realizar sus experimentos, ya que la presencia de otros puntos de acceso (AP) WiFi interfería con los experimentos. En general, se obtuvieron mejores resultados cuando se utilizaron los canales más bajos o más altos en la banda de $2,4 \mathrm{GHz}$. El número de AP en la banda de $5 \mathrm{GHz}$ era menor que en la banda de 2,4 GHz. Sin embargo, se apreciaba un peor rendimiento del HackRF One en 5 GHz. Teniendo en 


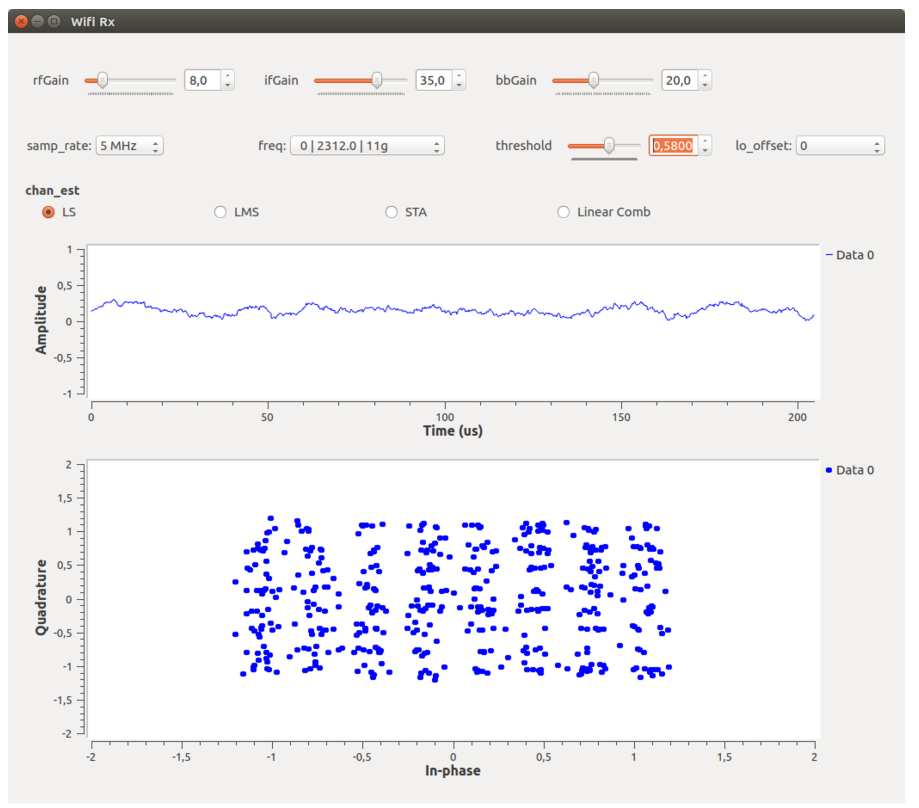

Fig. 2: Imagen de la interfaz gráfica del receptor. Los controles del receptor se colocan en la parte superior de la interfaz gráfica de usuario, la parte central muestra un gráfico de tiempo de la señal utilizada para determinar la llegada de un nuevo paquete. La parte inferior de la GUI corresponde a los símbolos recibidos en el último paquete. La imagen mostrada corresponde a una modulación 64-QAM.

cuenta este hecho y para evitar interferencias de los AP vecinos los experimentos se realizaron a $2,3 \mathrm{GHz}$.

\section{Liderazgo estudiantil}

Como se mencionó anteriormente, el papel del profesor en el ABP es presentar el proyecto y sugerir varias tareas destinadas a guiar la investigación de los estudiantes. Se espera que los estudiantes asuman progresivamente el liderazgo del trabajo. Esta parte de la metodología ABP duró la segunda y tercera sesiones de laboratorio, dependiendo del grado de autonomía de cada grupo de estudiantes. La actividad propuesta a los alumnos en esta etapa consistió en leer un archivo y enviarlo a través del canal inalámbrico para evaluar el porcentaje de errores de transmisión. El texto elegido fue el libro de caballería "El ingenioso hidalgo don Quijote de la Mancha", escrito por Miguel de Cervantes en 1605. La comparación entre los textos enviados y recibidos para distintas configuraciones del transmisor mostró que las diferencias se debían a las pérdidas de paquetes. Por lo tanto, para automatizar el análisis, el bloque wireshark connector disponible en el proyecto gr-ieee802-11 se utilizó para crear dos archivos de captura pcap, con paquetes enviados y recibidos, que fueron procesados posteriormente con un script Python para calcular la tasa de error. Las tasas de error logradas con cada modulación se muestran en Tabla 1. Se consideraron tres tamaños de paquetes diferentes para evaluar su contribución a la tasa de error. En general, la tasa de error aumentaba con el tamaño del paquete. El alto aumento de la tasa de error entre 16 QAM y 64 QAM sugiere que la calidad del canal no era lo suficientemente buena para trabajar con modulaciones 64 QAM, ya que la mayoría de los paquetes se perdieron en estos casos. 
Tabla 1: Tasa de error para las modulaciones disponibles considerando la nueva línea de varios tamaños de paquetes.

\begin{tabular}{lccc}
\hline \hline & \multicolumn{3}{c}{ Packet size (Bytes) } \\
Modulation & 500 & 1000 & 1500 \\
\hline BPSK 1/2 & 0.11 & 0.21 & 0.31 \\
BPSK 3/4 & 0.08 & 0.14 & 0.22 \\
QPSK 1/2 & 0.06 & 0.11 & 0.15 \\
QPSK 3/4 & 0.04 & 0.08 & 0.12 \\
16 QAM 1/2 & 0.06 & 0.11 & 0.11 \\
16 QAM 3/4 & 0.07 & 0.10 & 0.08 \\
64 QAM 2/3 & 0.60 & 0.85 & 0.90 \\
64 QAM 3/4 & 0.85 & 0.97 & 0.99 \\
\hline \hline
\end{tabular}

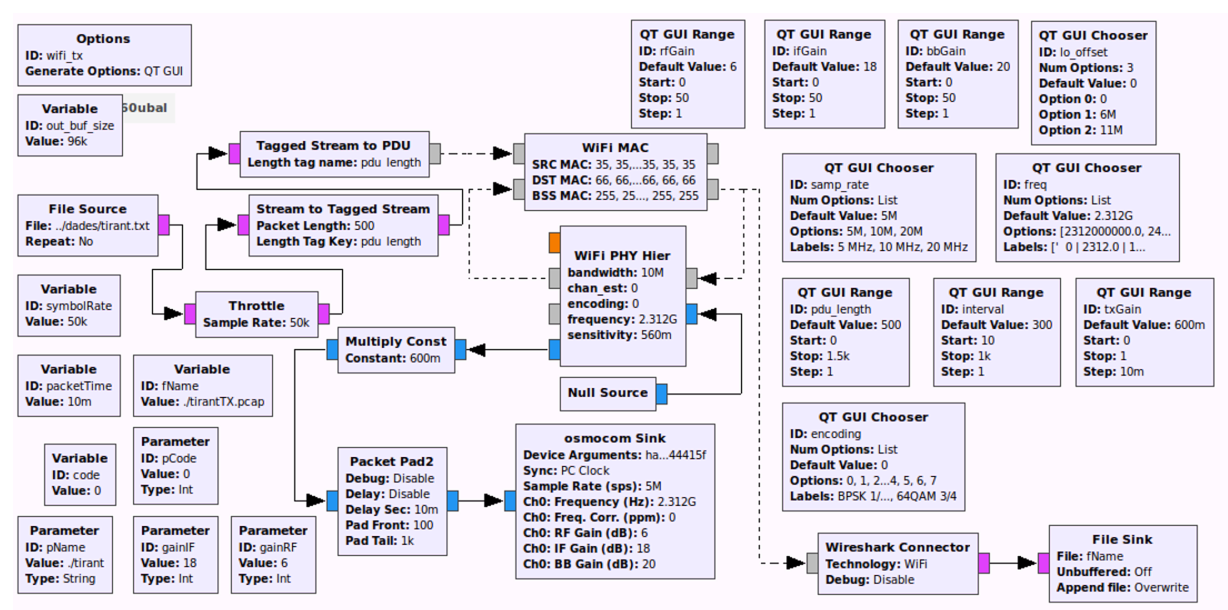

Fig. 3: Diagrama de bloques del transmisor propuesto a los estudiantes de GIT.

\section{Traslación del resultado de la experiencia ABP a los laboratorios en GIT}

La experimentación de ABP ha proporcionado al profesor información valiosa relacionada con el rendimiento del sistema y también con los problemas a los que se enfrenta. Toda esta información ha sido recogida y tenida en cuenta para diseñar una experiencia más guiada que se llevará a cabo en los próximos cursos académicos por parte de los estudiantes universitarios del grado GIT. La experiencia con los estudiantes de máster ha evidenciado la necesidad de empelar diferentes computadores para el transmisor y el receptor. Todos estos comentarios se han tenido en cuenta en la preparación de la experiencia que llevarán a cabo los estudiantes de grado.

La experiencia diseñada para estudiantes de grado consiste en un enfoque más guiado que la propuesta hecha a los estudiantes de máster. Los estudiantes de GIT recibirán los diagramas de bloques del transmisor y del receptor mostrados en la Fig. 3 y Fig. 4, respectivamente. Ambos diagramas incluyen el bloque wireshark connector para crear los archivos pcap. El script Python utilizado para analizar estos archivos pcap también se proporcionará a los estudiantes de GIT para que puedan centrarse en realizar la evaluación del sistema en lugar de diseñar el banco de pruebas. 


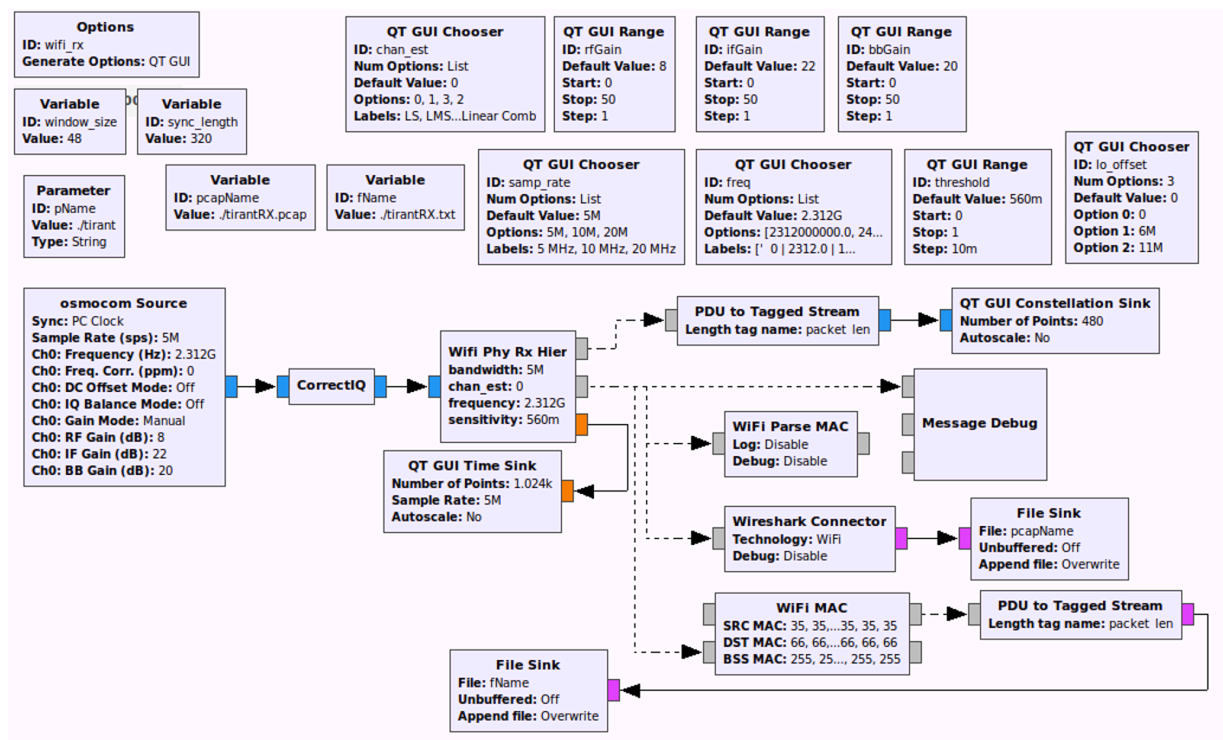

Fig. 4: Diagrama de bloques del receptor propuesto a los estudiantes de GIT.

\section{Resultados}

El resultado de la actividad basada en ABP planteada a los estudiantes del máster MITUV fue el informe con las instrucciones para el montaje del banco de pruebas y la realización de medidas. En general todos los grupos de trabajo completaron la experiencia con éxito. Aunque fue necesario corregir y adaptar el material que elaboraron para poder ser empleado por los estudiantes de grado. La principal ventaja que se ha observado en el caso de los estudiantes de máster es que la experiencia basada en ABP ha permitido que aquellos estudiantes que tenían más interés en la realización de la práctica pudiesen avanzar más y profundizar más la implementación del banco de pruebas. A la vez que aquellos que estaban menos interesados se limitaron a completar las actividades que se les plantearon. En cualquier caso todos resolvieron con éxito el problema que se les planteó.

En el caso de los estudiantes del grado GIT, se ha empleado una encuesta para cuantificar el impacto de esta experiencia en la implicación de los estudiantes en su formación. Para ello se ha adaptado una encuesta que evalúa el compromiso en el trabajo García-Ros y col. 2017; Schaufeli y Bakker 2004. Los estudiantes valoraron cada una de las 10 preguntas en una escala entre 1 (nunca) y 5 (siempre):

- Q1. En la universidad me siento lleno de energía.

- Q2. Pienso que las actividades son relevantes y significativas.

- Q3. El tiempo me parece que "vuela" cuando estoy estudiando.

- Q4. Me siento con gran fuerza y vigor mientras estudio.

- Q5. Me entusiasma lo que estudio. 
Tabla 2: Variación de los valores promedio de las respuestas de los estudiantes a cada una de las preguntas de la encuesta.

\begin{tabular}{lcccccccccc}
\hline \hline & Q1 & Q2 & Q3 & Q4 & Q5 & Q6 & Q7 & Q8 & Q9 & Q10 \\
\hline Previo & 2.69 & 3.33 & 2.41 & 2.47 & 3.33 & 2.92 & 2.86 & 2.33 & 3.11 & 3.19 \\
Posterior & 2.76 & 3.42 & 2.85 & 2.64 & 3.42 & 2.97 & 3.09 & 2.39 & 3.18 & 3.33 \\
Diferencia & 0.07 & 0.09 & 0.44 & 0.17 & 0.09 & 0.05 & 0.23 & 0.06 & 0.07 & 0.14 \\
\hline \hline
\end{tabular}

- Q6. Cuando estoy trabajando o estudiando me olvido de todas las cosas que pasan a mi alrededor.

- Q7. El trabajo en la universidad me resulta ilusionante.

- Q8. Tengo ganas de ir a clase cuando me levanto por la mañana.

- Q9. Me satisface trabajar con intensidad en mi carrera.

- Q10. En general, me siento muy satisfecho con mis estudios.

El laboratorio de FST en el que se llevó a cabo la experiencia está formado por dos partes. La primera parte se basa en simulaciones y cálculos de carácter teórico realizados en Matlab, mientras que en la segunda parte se plantean experiencias más aplicadas basadas en plataformas SDR. 36 estudiantes realizaron la anterior encuestas en dos ocasiones a lo largo del laboratorio para cuantificar cómo había contribuido la contribución la actividad llevada a cabo a su motivación. La primera encuesta se realizó al final del bloque de prácticas teóricas basadas en Matlab, y la segunda al final de curso tras haber realizado todas las prácticas basadas en SDR. El tiempo transcurrido entre ambas encuestas es de 2 meses, se ha considerado suficiente como para que en la realización de las encuestas por segunda vez los estudiantes no recuerden lo que contestaron en la primera ocasión.

En los resultados mostrados en Tabla 2 se presentan las puntuaciones promedio de los estudiantes a cada una de las preguntas. Cabe destacar que la valoración promedio de todas las preguntas es ligeramente mayor tras haber realizado la actividad propuesta respecto a la encuesta previa. Las diferencias de valoración más significativas se dan en las preguntas 3,7 y 4. También se aprecia una mayor satisfacción de los estudiantes con los estudios que realizan.

La última de las preguntas planteadas en la encuesta está relacionada con la satisfacción general de los estudiantes. Las 9 preguntas restantes evalúan 3 aspectos relacionados con la implicación académica:

- Energía: es un aspecto relacionado con la capacidad de resiliencia del alumno en la resolución de problemas. (Q1, Q4, Q8)

- Absorción: evalúa la capacidad del alumno para concentrarse en aquellas tareas que está realizando. (Q3, Q6, Q9)

- Dedicación: evalúa la percepción del estudiante sobre la relevancia de las actividades que realiza. (Q2, Q5, Q7) 
Tabla 3: Variación de los valores promedio de la energía, absorción e implicación de los estudiantes.

\begin{tabular}{lccc}
\hline \hline & Energía & Absorción & Dedicación \\
\hline Previo & 2.50 & 2.82 & 3.17 \\
Posterior & 2.60 & 3.00 & 3.31 \\
Diferencia & 0.1 & 0.18 & 0.14 \\
\hline \hline
\end{tabular}

Para cuantificar cada uno de los aspectos relacionados con la motivación académica se han agregado las respuestas a todas las preguntas relacionadas con cada uno de ellos. En la Tabla 3 se presenta la valoración de los estudiantes en cada uno de los aspectos en la encuesta previa y posterior, así como la diferencia en la valoración. Se aprecia una ligera mejora en todos los aspectos desde la primera a la segunda encuesta. El aspecto en el que se aprecia una mayor mejora es en la capacidad del estudiante en centrarse en la realización de las tareas que está llevando a cabo.

\section{Conclusiones}

La experiencia previa de los autores reveló la demanda de los estudiantes de sesiones de laboratorio de Comunicaciones Digitales y Procesado de Señales para Comunicaciones más orientadas a las aplicaciones. En este sentido, las plataformas SDR ha demostrado ser unas excelentes herramientas para introducir aplicaciones reales en la enseñanza de las Comunicaciones Digitales. Este trabajo presenta la experiencia de aprendizaje basada en proyectos realizada con alumnos del MITUV de la ETSE-UV, donde se les propuso diseñar un banco de pruebas para evaluar el rendimiento de un sistema de transmisión WiFi. Esta actividad de aprendizaje activo contribuyó a posicionar a los estudiantes en el centro de su proceso de aprendizaje. La principal ventaja que se ha observado con los estudiantes del MITUV es que la experiencia basada en ABP ha proporcionado más libertad a los estudiantes para profundizar en la realización de las prácticas en función de sus intereses.

A partir de los resultados obtenidos con el trabajo llevado a cabo por los estudiantes de máster, se han preparado unas experiencias más guiadas que han sido realizadas por los estudiantes de grado. En el caso de los estudiantes de grado se ha cuantificado el impacto del uso de plataformas SDR en la implicación en su formación académica. La encuesta empleada para evaluar la implicación de los estudiantes contempla 3 aspectos: energía, absorción y dedicación. El impacto de la realización de experiencias basadas en SDR ha contribuido de forma positiva en los tres aspectos mencionados. Siendo el más relevante el caso de la mejora en la absorción, o capacidad del estudiante para centrarse en la tarea que está haciendo en ese momento. 


\section{Referencias bibliográficas}

Bloessl, B. y col. (2018). "Performance assessment of IEEE 802.11p with an open source SDR-based prototype". En: IEEE Transactions on Mobile Computing 17 (5), págs. 1162-1175.

El-Hajjar, M. y col. (2014). "Demonstrating the practical challenges of wireless communications using USRP". En: IEEE Communications Magazine 52.5, págs. 194-201. ISSN: 0163-6804. DOI: 10.1109/MCOM. 2014.6815912.

ETSI (2009). ETSI EN 300744:2009. "Digital Video Broadcasting (DVB): Framing structure, channel coding and modulation for digital terrestrial television". http: //www.etsi.org/deliver/etsi_en/300700_300799/300744/01.06.01_60/ en_300744v010601p.pdf (Visited on: 27/02/2019).

García-Ros, Rafael y col. (2017). "The Schoolwork Engagement Inventory: Factorial Structure, Measurement Invariance by Gender and Educational Level, and Convergent Validity in Secondary Education (12-18) Years". En: Journal of Psychoeducational Assessment 36 (6), págs. 588-603. DOI: 10.1177/0734282916689235.

IEEE (2016). IEEE Standard for Information technology Telecommunications and information exchange between systems Local and Metropolitan area networks. Specific requirements. Part 11: Wireless LAN Medium Access Control (MAC) and Physical Layer (PHY) Specifications.

Jimenez, V. P. G. y col. (2017). "Learning Mobile Communications Standards through Flexible Software Defined Radio Base Stations". En: IEEE Communications Magazine 55.5, págs. 116-123. ISSN: 0163-6804. DOI: 10.1109/MCOM.2017.1601219.

Mitola, J. (1992). "Software radios-survey, critical evaluation and future directions". En: Proc. IEEE National Telesystems Conference, (NTC'92). Washington DC, USA, May, págs. 15-23.

Schaufeli, Wilmar y Arnold Bakker (2004). UWES: Utrecht Work Engagement Scale. Inf. téc. Utrecht University.

Segura, J. y col. (2016). "Innovación en docencia de sistemas de comunicación en el Grado de Ingeniería Telemática de la UVEG". En: Proceedings of the 20 Congreso de Innovación Educativa y Docencia en Red (In-Red 2016). Jul 7-8, Valencia (Spain).

- (2017). "Uso de software defined radio en la enseñanza de sistemas de telecomunicaciones". En: Actas de las XIII Jornadas de Ingeniería Telemática (Jitel 2017). Sept, Valencia (Spain), págs. 370-378.

Stewart, R. W. y col. (2015). "A low-cost desktop software defined radio design environment using MATLAB, simulink, and the RTL-SDR". En: IEEE Communications Magazine 53.9, págs. 64-71. ISSN: 0163-6804. DOI: 10.1109/MCOM. 2015. 7263347. 